\title{
El uso de las TIC en el currículo de inglés de Edu- cación Primaria por parte del profesorado novel
}

\author{
Juan Antonio SOLÍS BECERRA ${ }^{1}$ \\ Isabel M. SOLANO FERNÁNDEZ ${ }^{2}$ \\ Universidad de Murcia \\ ${ }^{1}$ Departamento de Didáctica de la Lengua y la literatura \\ ${ }^{2}$ Departamento de Didáctica y Organización Escolar \\ 1jasolis@um.es / 2imsolano@um.es
}

Recibido: agosto 2012

Aceptado: enero 2013

\section{RESUMEN}

Este trabajo presenta un estudio cuyo objetivo principal es analizar el uso que de las TIC están haciendo en el aula los maestros «noveles» de inglés, es decir, los que finalizaron sus estudios el año anterior al de su incorporación como docentes. Pero este estudio gira alrededor de un eje central, que es el propio Currículo Oficial del área de Inglés en Educación Primaria de la Región de Murcia y de la actuación de los maestros «noveles» de conformidad con éste. Pretendemos así encontrar resultados que nos sirvan de guía para detectar las principales necesidades formativas de los docentes.

Palabras clave: Tecnologías de la Información y la Comunicación (TIC), enseñanza del inglés, Enseñanza de Lenguas Asistida por Ordenador (ELAO), competencias, educación primaria.

The use of ICT across the English area curriculum for Primary Education by novice teachers

\begin{abstract}
This paper presents a study whose main objective is to analyse the use of Information and Communication Technologies (ICT) by those teachers who finished their degrees as English primary teachers just one year before starting to work at schools. This study focuses on the official curriculum for the area of English language in primary education in the Region of Murcia, as well as on the practice developed by these teachers according to the requirements of the curriculum. In this line, we intend to find useful results for identifying correctly teaching needs in this area.

Keywords: Information and Communication Technologies (ICT), English teaching, Computer Assisted Language Learning (CALL), competences, primary education.
\end{abstract}


L'utilisation des TIC par les nouveaux instituteurs dans le curriculum d'anglais de l'Education Primaire

\section{RESUMÉ}

Ce travail présente une étude dont l'objectif principal est celui d'analyser l'usage des TIC que font en classe les instituteurs de la spécialité d'anglais (L.E), qui ont fini leurs études l'année précédente et qui travaillent actuellement. Mais cette étude est centrée sur un axe qui n'est autre que le curriculum officiel de l'aire d'anglais (L.E) dans l'Education Primaire de la Région de Murcie. De cette façon nous prétendons analyser, d'une part, le rôle que ce curriculum accorde aux TIC dans cette aire et, d'autre part, le rôle des nouveaux instituteurs en conformité avec le curriculum; nous prétendons ainsi arriver à des résultats qui nous serviraient de guide pour détecter les principales nécessités formatives dans ce champ.

Mots-clé: Technologies de l'information et de la communication (TIC), enseignement d'anglais, Enseignement des langues assistées par ordinateur (ELAO), compétences, éducation primaire.

SUMARIO: 1. Introducción. 2. Metodología. 2.1. Muestra. 2.2. Objetivos de la investigación. 2.3. Recogida de información e instrumento. 3. Resultados. 3.1. Resultados referidos al primer ciclo de Primaria. 3.2. Resultados referidos al segundo ciclo de Primaria. 3.3. Resultados referidos al tercer ciclo de Primaria. 4. Conclusiones y discusión. 5. Referencias bibliográficas.

\section{INTRODUCCIÓN}

La preocupación por la formación de los docentes en cuestiones referidas a la integración curricular de las TIC es una constante desde hace décadas. Autores como Cabero (2004), Martínez (2007), Area (2007a y 2007b), Solano (2010), y en su orígenes, autores como Cuban (1986), Clark y Salomon (1986) y Clark (2001), plantearon la necesidad de repensar la innovación tecnológica desde la vertiente más pedagógica, enfatizando así entre otros aspectos, los elementos del curriculum y la formación del profesorado en aspectos instrumentales y metodológicos. Recientemente, el modelo didáctico Tpack ${ }^{1}$ (Technological Pedagogical Content Knowledge) ha repensado el diseño de actividades con TIC, considerando que debemos poner tres conocimientos expertos: el disciplinar, el tecnológico y el pedagógico, de cuya intersección se derivan otros tipos de conocimientos que culminan en el Conocimiento pedagógico disciplinar tecnológico, es decir, el dominio de tecnologías específicas adecuada a la enseñanza de una disciplina en particular (Harris y Hofer, 2009; Koehler y Mishra, 2009).

En este trabajo, presentamos un referido a la enseñanza de idiomas en educación primaria, y más concretamente en el caso del inglés. En este trabajo reflexio-

${ }^{1}$ Página web del modelo Tpack: http://www.tpck.org/ 
naremos sobre el uso que los docentes noveles de inglés realizan de las TIC atendiendo al Currículum Oficial de Primaria en la Región de Murcia.

En los últimos años, y con más precisión en la última década, han proliferado las investigaciones cuyo objeto de estudio son las tecnologías en la enseñanza de idiomas. En este contexto cabe destacar lo que en inglés se denomina ComputerAssisted Language Learning (CALL). La proliferación de estudios desde esta perspectiva se debe, en gran medida, a las nuevas posibilidades que ofrecen las TIC y especialmente la red Internet.

Warshauer y Healey (1998) nos advierten de que los ordenadores comenzaron a ser utilizados en la enseñanza de lenguas a partir de los años 60. Estos autores distinguen tres fases en la evolución de la tecnología. En primer lugar la fase «behaviorista», desarrollado entre los años 60 y 70, que respondía al planteamiento por repetición. La segunda fase es la llamada "comunicativa», que surgió a finales de los años 70 y principios de los 80 , y responde a las teorías cognitivas que entienden el aprendizaje como un proceso de descubrimiento, creatividad y desarrollo. No será hasta finales de los años 80 y principios de los 90 cuando podamos hablar de la aparición del modelo «integrador», de corte constructivista, que aboga por el uso del lenguaje en contextos sociales auténticos. Así, nos vamos a encontrar con acercamientos desde la perspectiva del enfoque por tareas, el aprendizaje basado en proyectos o la aproximación que se basa en el contenido, y que ha sido analizado y experimentado por autores como Curran, Deguent, Luna, Mileto y Van der Straeten (2000), Ellis (2003), Delfín, Dettori y Lupi (2009) y Martín López (2009)

Desde finales de la década de los 90 el desarrollo de la tecnología CALL ha sido imparable, al igual que lo han sido los estudios que centran su ámbito de investigación en el uso de estas tecnologías por parte de profesores de idiomas (Hubbard y Levy, 2006; Kassen, Lavine, Murphy-Judy y Peters, 2007). Pero Beatty (2003, p.14) afirma que se ha producido un cambio en el centro de atención de las investigaciones centradas en CALL, ya que se ha pasado de considerar en un primer momento si había que utilizar o no las nuevas tecnologías en la enseñanza de idiomas, a centrarse en ver cómo deberían utilizarse y con qué propósito, como dicen Hubbard y Levy (2006, p. 11): «necesitan saber porqué hacen lo que hacen». Igualmente, y muy vinculado a lo que acabamos de decir, hay áreas que todavía en la actualidad necesitarían ser más estudiadas, como por ejemplo si la formación previa en tecnología educativa de los maestros les sirve para impulsar el uso de estas tecnologías en sus propias aulas (Hong, 2010). De aquí que las últimas preocupaciones de los investigadores del aprendizaje de segundas lenguas se estén centrando en este último aspecto.

Siguiendo este análisis, nos planteamos si la clave puede estar en la formación inicial del profesorado, y por tanto, en la configuración de asignaturas y/o contenidos que atienda al uso de las TIC en el aula de inglés, o en la formación continua. De acuerdo con el objetivo de este estudio, nos centraremos en reflexionar sobre la formación inicial del profesorado. Generalmente la asignatura que se dedica en los 
títulos de formación de maestros de lenguas extranjeras vinculada al uso de las nuevas tecnologías es de último curso o de posgrado, pero en cambio Hegelheimer (2006) apoya que la formación al respecto venga ya desde el principio de los estudios universitarios para así poderlo aplicar a otras asignaturas del plan de estudios de su titulación como docentes. Hay autores que se muestran cautos respecto a la introducción de sólo una o dos asignaturas o relacionados con CALL en los programas de formación del profesorado (Desjardins y Peters, 2007; Kessler, 2006; Luke y Britten, 2007).

En el contexto español, los planes de estudios ya extinguidos (Diplomado en Magisterio) contemplaban una asignatura cuatrimestral en tercer curso orientada a aprender la aplicación didáctica de las TIC. En cuanto a los planes actuales, el Grado en Educación Primaria, en la que se incluye la especialización de inglés, contempla las TIC como un contenido transversal que se estudió en los primeros cursos, con lo cual las asignaturas propias de la especialización, podrán aprovecharse de los contenidos instrumentales y de aplicación didáctica de las TIC ya aprendidos en estos primeros años. Esto permitirá que los futuros formadores o formadores que ya se encuentran trabajando adquieran las denominadas «technopedagogical skills» (Desjardins y Peters, 2007), o como dirían Tochon y Black (2007, p.296) «technopedagogy» para hacer alusión a «la integración apropiada de la tecnología de un modo pedagógico»».

\section{METODOLOGÍA}

La metodología planteada en esta investigación para el logro de los objetivos es de corte no experimental. El enfoque metodológico utilizado para esta investigación ha sido el empírico-analítico ya que hemos procedido a la recogida de información fragmentada y a su posterior medición para analizar la realidad objeto de estudio. Para realizar el tratamiento estadístico de los datos, estos fueron sometidos a un proceso de codificación y categorización, permitiendo así su interpretación y la elaboración de conclusiones.

\subsection{Muestra}

La población que hemos seleccionado para nuestro trabajo ha sido la de aquellos titulados que finalizaron la diplomatura de Magisterio en Lenguas Extranjeras (Inglés) en el curso 2009/2010 en la Universidad de Murcia, y que se encontraban trabajando como docentes en Educación Primaria durante el curso 2010/2011.

La muestra invitada quedó conformada por 31 alumnos que habían finalizado sus estudios de Maestro en Lenguas Extranjeras (Inglés) en la Universidad de Murcia, 21 alumnos que superaron la asignatura en junio de 2009 y 10 que finalizaron en septiembre del mismo año. A continuación tuvimos que ponernos en contacto con ellos para conocer su situación laboral. Nuestra muestra tenía un objetivo muy claro: solamente podían ser objeto de estudio aquellos que se dedicaran a la docencia de la lengua inglesa en el nivel educativo de Educación 
Primaria, por lo que no serían nuestro objeto de investigación los que sólo dieran clase en otras etapas educativas o cursos inmersos en Programas de Bilingüismo, o los que, aún dando clase en primaria impartieran docencia sobre otras áreas del Currículo. Nos encontramos con los siguientes resultados: 5 no trabajan porque se están dedicando a ampliar su formación; 1 trabaja en otro sector; 2 se dedican exclusivamente a la docencia en Educación Infantil; 1 trabaja como maestra pero en otra comunidad autónoma y por tanto no es objeto de estudio al tener un Currículo oficial diferente; y 1 se dedica a la docencia en primaria pero no como especialista de inglés.

A partir de estos resultados la muestra productora de datos quedó conformada por 21 profesores noveles. No obstante 3 no quisieron participar, pero a pesar de todo la muestra final supone una representación de casi un $90 \%$ de la muestra invitada. La población quedó distribuida de la siguiente forma (Figuras 1 y 2)

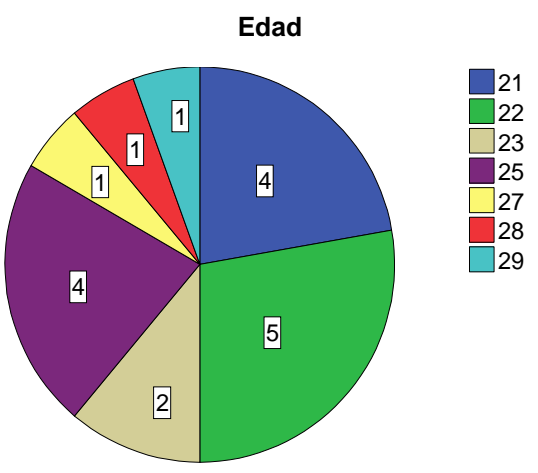

Figura 1: Edad de los sujetos de la muestra.

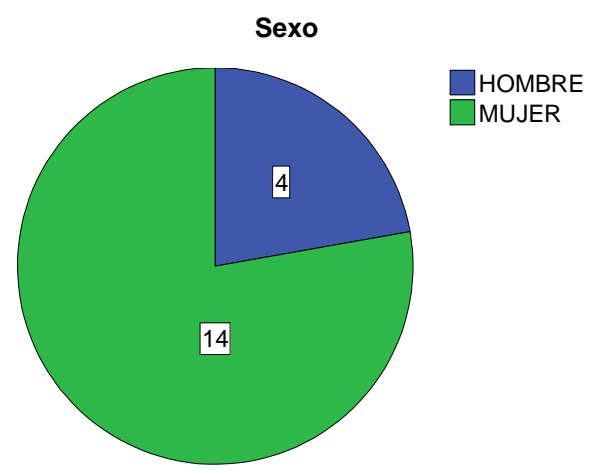

Figura 2: Edad de los sujetos de la muestra

\subsection{Objetivos de la investigación}

El propósito de esta investigación fue analizar el uso que hacen de las TIC los maestros noveles de inglés en sus respectivas aulas, prestando especial atención al cumplimiento de los principios establecidos al respecto en el currículo oficial del área de inglés de Educación Primaria en la Región de Murcia. Es preciso indicar que se optó por el curriculum oficial de esta Comunidad porque los sujetos de la muestra estaban desempeñando su labor educativa en ella, pero las coincidencias por el Decreto de Enseñanza Mínimas nacional es elevado. Para conseguir este propósito, planteamos los siguientes objetivos:

1) Identificar aquellos puntos en los que el Currículo hace mención al uso de las TIC.

2) Conocer el grado de competencia informática de los maestros noveles de inglés de la Región de Murcia. 
3) Analizar la formación específica que los maestros noveles tienen en relación con las Tecnologías de la Información y la Comunicación en la enseñanza de idiomas.

4) Analizar la intervención e innovación docente con TIC que los maestros noveles realizan en las aulas en las que están ejerciendo su actividad didáctica.

Por una cuestión de espacio, en este trabajo vamos a centrarnos en los objetivos 1 y 4. Los dos restantes han sido expuestos en otras publicaciones.

\subsection{Recogida de información e instrumento}

El instrumento utilizado para la recogida de datos fue un cuestionario estructurado en diversos bloques que atendían a cada uno de los aspectos que queríamos analizar. En este trabajo, por limitación de espacio, nos vamos a centrar exclusivamente en aquellos datos relacionados con la práctica docente del profesorado y el uso que hace de las TIC en el aula, utilizando como parámetros de referencia los postulados del Currículo para esta área. Estos puntos quedan reflejados en los Bloques V y VI del cuestionario. El Bloque V estaba orientado a obtener información sobre las TIC en el Currículo de Inglés en Educación Primaria. Este apartado está dividido a su vez en tres subapartados, cada uno corresponde a los tres ciclos de Educación Primaria, teniendo en cuenta las competencias y contenidos que el $\mathrm{Cu}$ rrículo Oficial establece, comprobando así el grado de correlación entre lo establecido en el currículo y lo que realizan los docentes. El Bloque VI hace referencia a la frecuencia de uso de las TIC.

El cuestionario fue sometido a validación de expertos, mediantes un proceso de triangulación entre expertos de Didáctica de la Lengua y la Literatura (2) y un experto en Tecnología Educativa (1).

A los profesores del estudio se les facilitaron dos opciones de cumplimentación y devolución del cuestionario: (a) por correo electrónico y (b) por correo postal. No obstante, el 100\% del profesorado participante eligió la opción del correo electrónico.

El cuestionario se encuentra publicado para su difusión y descarga en el repositorio institucional de la Universidad de Murcia ${ }^{2}$.

\section{RESULTADOS}

En este apartado vamos a analizar los resultados referidos al uso que el profesorado novel de inglés de Educación Primaria realiza de las TIC. Para una mejor observación de los datos los vamos a clasificar por ciclos, pero antes de detenernos en

\footnotetext{
${ }^{2}$ Cuestionario para evaluar el uso de las TIC en maestros de inglés en Educación Primaria que finalizaron su titulación universitaria en junio y septiembre de 2010:

http://digitum.um.es/xmlui/handle/10201/30145
} 
cada uno de ellos, merece la pena resaltar que sólo el $50 \%$ de los encuestados responde afirmativamente al ítem referido a si conocen el tratamiento que el Currículo de la Región de Murcia de Educación Primaria otorga a las TIC en el área de inglés. Un análisis más exhaustivo sobre cómo el Currículo que mencionamos recoge el papel de las TIC se puede consultar en Solís y Macías (2009), estudio que tomamos como referencia para este artículo.

\subsection{Resultados referidos al primer ciclo de Primaria}

La muestra que vamos a analizar en este bloque está integrada por 11 de los encuestados y se corresponde exclusivamente con aquellos docentes que han dado clase durante este curso académico en este ciclo, aunque no necesariamente de manera exclusiva. El $27 \%$ de los encuestados admite no utilizar con frecuencia las TIC en este ciclo.

Nos centraremos en primer lugar en la destreza listening, que es la más valorada por el Currículo en cuanto al uso de las TIC. El 82\% de los alumnos afirma utilizar la lectura en voz alta como recurso. En este sentido, el $45 \%$ reconoce que tampoco usa con frecuencia grabaciones audiovisuales para esta destreza (Figura 3).

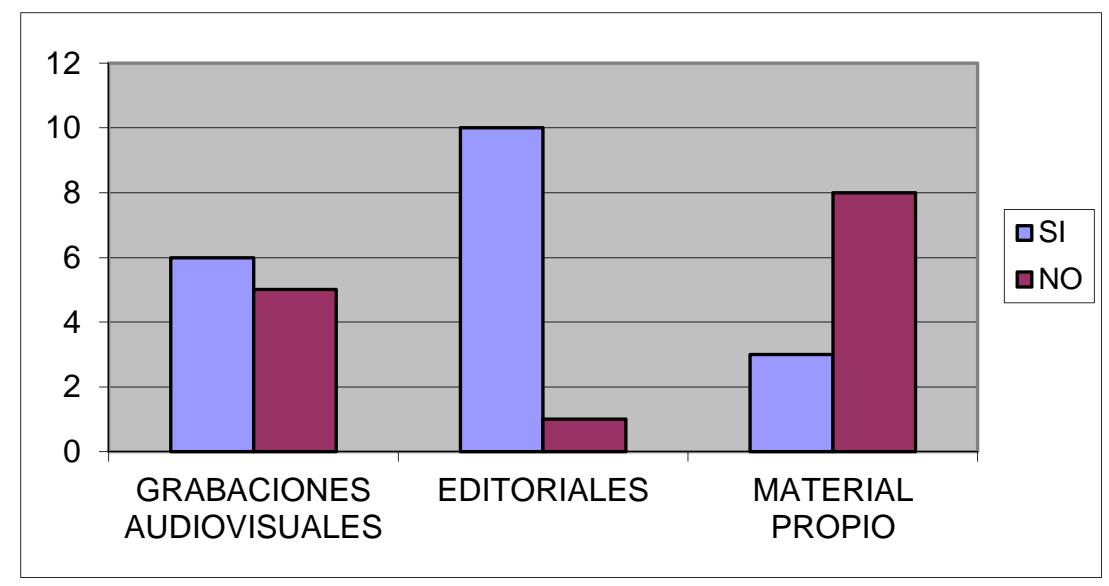

Figura 3: Origen del material para la destreza listening $\left(1^{\circ} \mathrm{Ciclo}\right)$.

Cuando utilizan grabaciones digitales vemos que prácticamente la totalidad recurre al material proporcionado por las editoriales, de hecho sólo un $9 \%$ de los encuestados reconoce no utilizarlo. El 27\% de los encuestados crean su propio material para esta actividad (Figura 3).

En cuanto al soporte del material para la destreza listening que utilizan en el aula vinculado al uso de las TIC, tan sólo un maestro utiliza las cintas tradicionales. De hecho, el $100 \%$ utiliza como soporte los CD. El siguiente soporte digital más utilizado por frecuencia de uso es el DVD, que lo utilizan el $72 \%$ de los encuesta- 
dos. Finalmente conviene resaltar que el $45 \%$ recurre a Internet para buscar recursos para esta destreza (Figura 4)

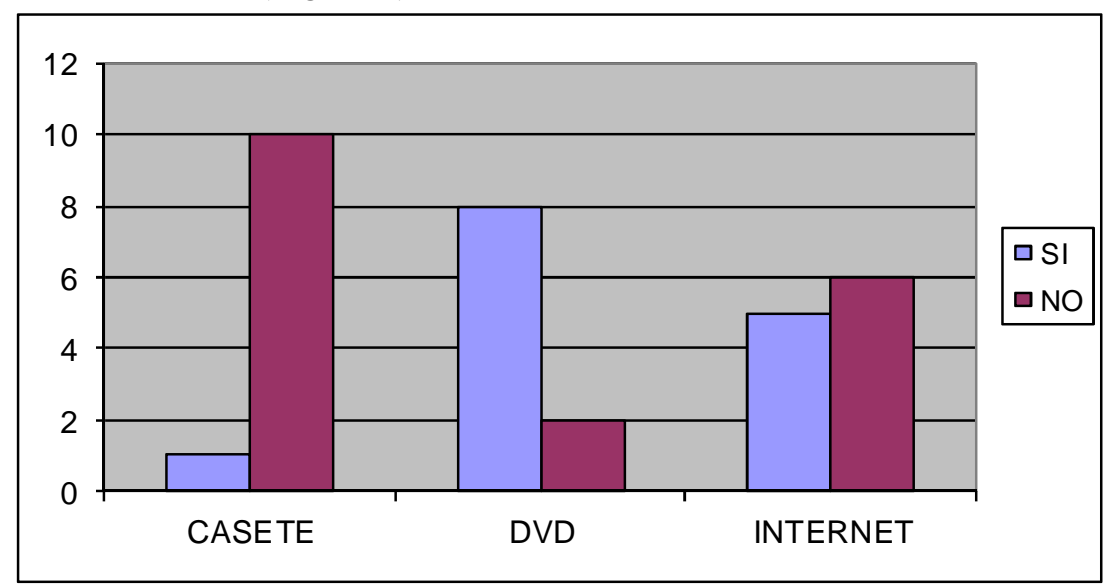

Figura 4: Soporte para el material utilizado en la destreza listening $\left(1^{\circ}\right.$ Ciclo $)$.

Todos los encuestados por unanimidad reconocen que la principal finalidad de las actividades que planifican para la destreza listening es para que los alumnos comprendan la información, en lugar de la repetición de la misma.

Otro de los criterios establecidos en el Currículo es que la introducción del uso de las TIC sea de manera progresiva. A este respecto el $45 \%$ de los encuestados reconoce no hacerlo de este modo.

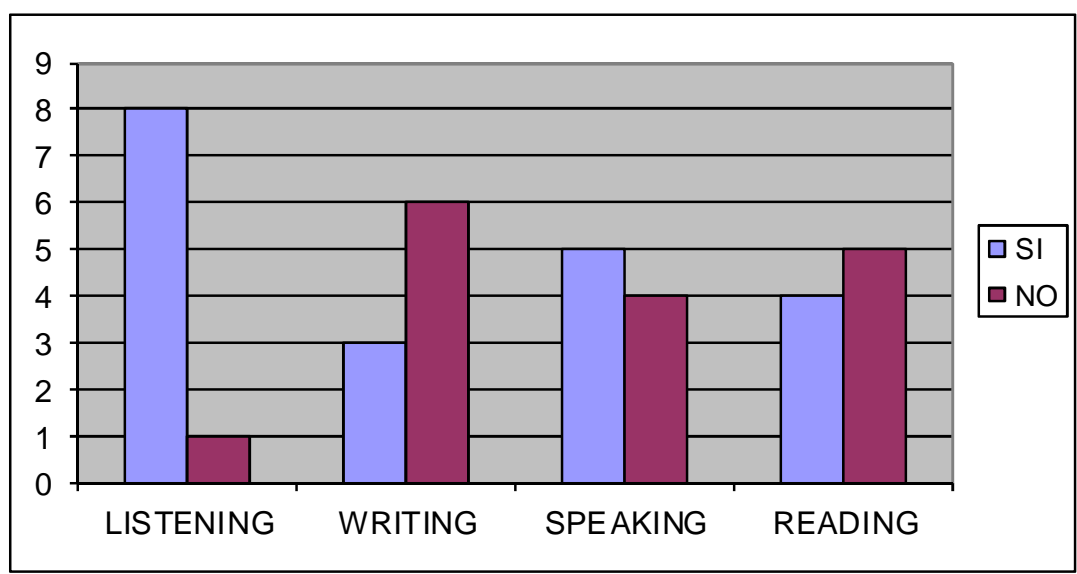

Figura 5: Uso de las TIC por destrezas comunicativas $\left(1^{\circ}\right.$ Ciclo $)$.

En la Figura 5 comprobamos que la destreza listening es aquella en la que más utilizan los docentes las nuevas tecnologías, a ésta le sigue la destreza speaking, en la que lo hacen algo más de la mitad de los encuestados (55\%). Tenemos que seña- 
lar que el instrumento más utilizado para esta destreza es el podcast. En las dos destrezas restantes, por su propia naturaleza y las características del ciclo al que estamos haciendo alusión, son menos los encuestados que recurren al uso de las TIC, y especialmente, en el caso de writing, cuyo porcentaje es de un $66 \%$.

\subsection{Resultados referidos al segundo ciclo de Primaria}

Éste es el ciclo en el que participa el mayor número de encuestados: son un total de 13. Nos detendremos a continuación en la destreza listening. En la Figura 6 podemos comprobar que prácticamente la totalidad de los encuestados $(92 \%)$ utiliza materiales multimedia para las actividades en las que se ve implicada esta destreza en el aula. En cambio, y a diferencia a la situación observada en el primer ciclo es un elevado número de docentes (92\%) el que no crea su propio material para trabajar esta destreza. En estas actividades de listening son un elevado número de encuestados los que no recurre a la red Internet, un $77 \%$. Tenemos que recalcar además que el $100 \%$ de los encuestados reconoce utilizar el material suministrado por las editoriales, de ahí la escasa intervención en creación y búsqueda de material que estamos apreciando. El Currículo manifiesta expresamente que en este ciclo las actividades de listening con nuevas TIC deben permitir desarrollar la capacidad de extraer información. De los datos obtenidos observamos que el $84 \%$ de los encuestados cumplen esta finalidad.

En la Figura 7 hemos reflejado los principales datos vinculados a la actuación de los docentes relacionada con la destreza reading. Todos los encuestados responden que ya no utilizan única y exclusivamente el formato papel para que sus alumnos realicen actividades relacionadas con la lectura. De todas formas, son más los encuestados que responden negativamente al uso de productos digitales en la destreza lectura, un 53\%. Cuando son interrogados respecto a los equipos que utilizan para realizar actividades de lectura en formato digital, los pocos que lo utilizan para este fin (38\%), lo hacen en los equipos del centro, mientras que al mencionar los equipos personales en el hogar familiar sólo el $15 \%$ responde que sus alumnos lo utilizan. También destaca el hecho de que sólo el 15\% de los encuestados utilice la pizarra digital interactiva (PDI) para este fin, aunque quizás en este caso deberíamos responder a la disponibilidad real de PDI que existe en los centros, y específicamente, en las aulas, así como en la formación que el profesorado tiene para usarla.

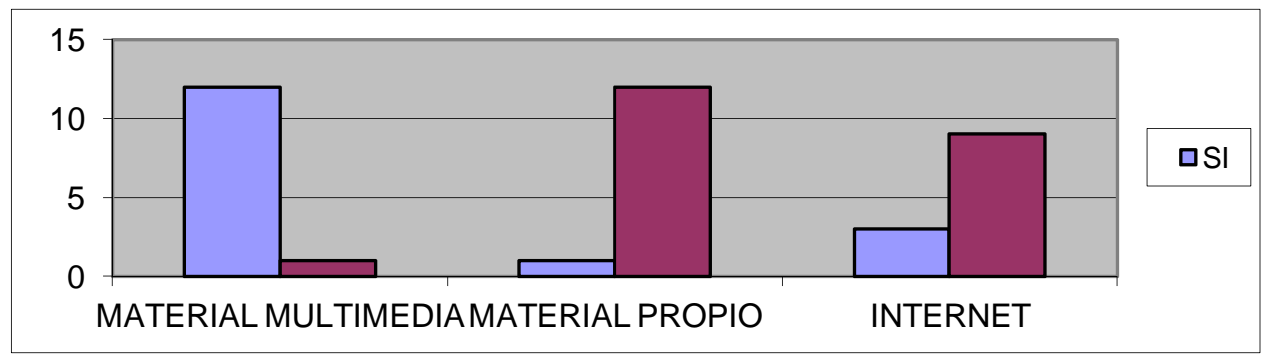

Figura 6: TIC en la destreza listening $\left(2^{\circ}\right.$ Ciclo $)$. 


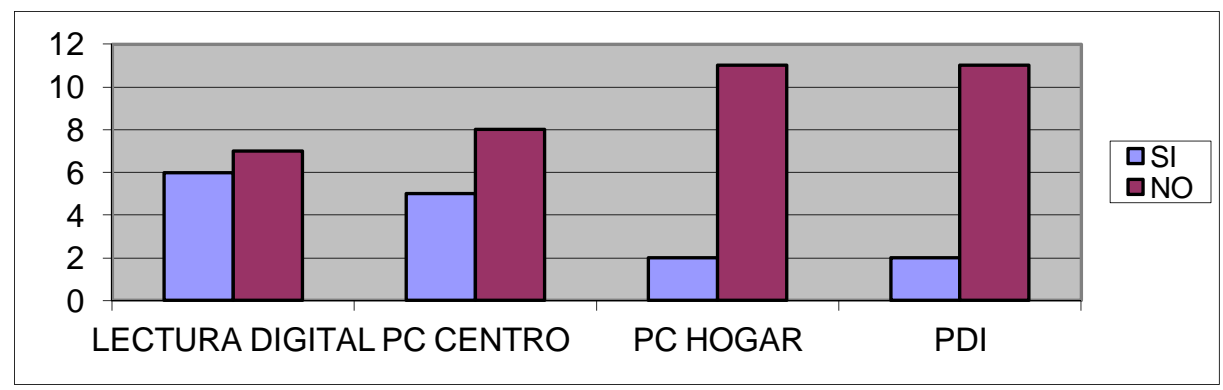

Figura 7: Soportes de lectura digital $\left(2^{\circ}\right.$ Ciclo $)$.

Respecto al material de lectura digital utilizado, algo más de la mitad de los encuestados (53\%) reconocen que sus alumnos utilizan Internet para la lectura de información. Sin embargo, el 92\% de los encuestados afirma utilizar como lecturas el material suministrado por las editoriales.

La Figura 8 refleja los datos relacionados con la destreza escritura. Recordemos que en este ciclo la exigencia por parte del Currículo es mayor. De todas formas podemos comprobar que el 53\% de los encuestados afirman que sus alumnos utilizan las TIC para escribir información, pero el número es menor cuando los encuestados reconocen que sus alumnos utilizan las TIC para transmitir información (hacemos esta distinción que también recoge el Currículo), de hecho supone el 23\% los que lo hacen. En cambio, al hablar de comunicarse, el 30\% reconoce que sus alumnos lo utilizan con esta finalidad.

A continuación nos vamos a centrar en el uso de las TIC para fomentar el aprendizaje autónomo del alumno. El 53\% de los encuestados reconoce abiertamente no llevarlo a cabo en la práctica. Cuando hablamos del uso de las TIC para que el profesor fomente el refuerzo de la lengua extranjera de sus alumnos tanto en clase como de forma autónoma, las respuestas rozan el 50\% para ambos casos. En cambio, cuando les interrogamos para saber si utilizan las TIC para que los alumnos amplíen el conocimiento de la lengua extranjera de forma autónoma, el $56 \%$ responde negativamente.

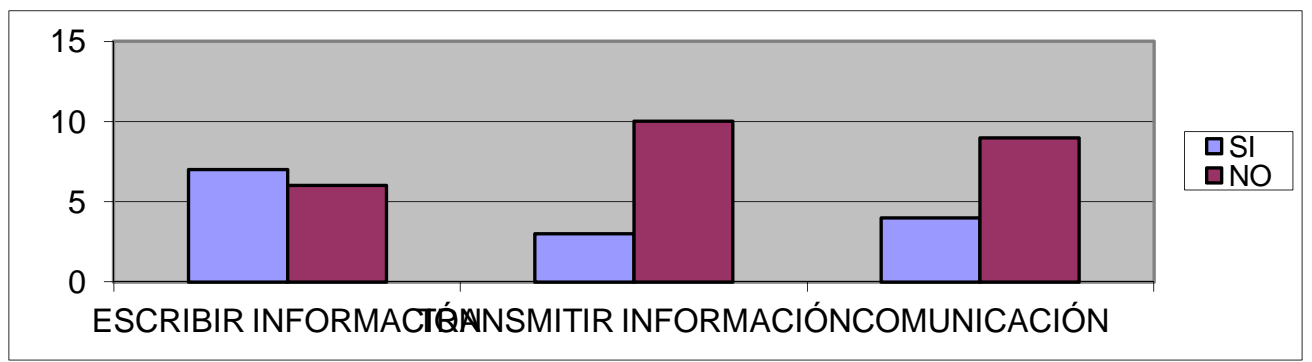

Figura 8: Finalidad de la escritura en soporte electrónico $\left(2^{\circ}\right.$ Ciclo). 
Respecto a los procesos cognitivos implicados en el tratamiento de la información exigido por el Currículo mediante las TIC destaca el alto número de docentes que reconocen que sus alumnos no utilizan las TIC para recuperar información sobre temas conocidos, un $83 \%$. Sin embargo, cuando a la búsqueda de información sobre estos mismos temas se refiere esta cifra disminuye hasta un 58\%. Entre los resultados encontramos además que el $75 \%$ reconozca que sus alumnos no utilizan las TIC para recopilar información.

\subsection{Resultados referidos al tercer ciclo de Primaria}

En este ciclo participan menos docentes encuestados, ya que suponen el $44 \%$ de la muestra $(\mathrm{n}=8)$. Centrándonos en los datos relacionados con la destreza listening vemos que prácticamente todos $(87 \%)$ afirman utilizar grabaciones multimedia. Sin embargo, sólo un $12 \%$ reconoce que crea su propio material para esta destreza. Una vez más, y al igual que ocurría en el ciclo anterior los encuestados responden por unanimidad que utilizan el material que les ofrecen las editoriales. El 62\% reconoce, además, utilizar para las actividades de listening material extraído de Internet.

El Currículo señala procesos cognitivos que deben realizar los alumnos con las actividades de listening utilizando las TIC: escuchar, comprender, extraer, contrastar y comprobar información (Figura 9). Las dos primeras sí son utilizadas por la totalidad de los encuestados. Cuando hablamos de extraer información un $12 \%$ no lo hace. Pero las cifras se invierten en los dos últimos, en especial el de contrastar la información, que sólo un $12 \%$ reconoce realizarlo. Respecto a comprobar la información lo hace el $50 \%$ de los encuestados.

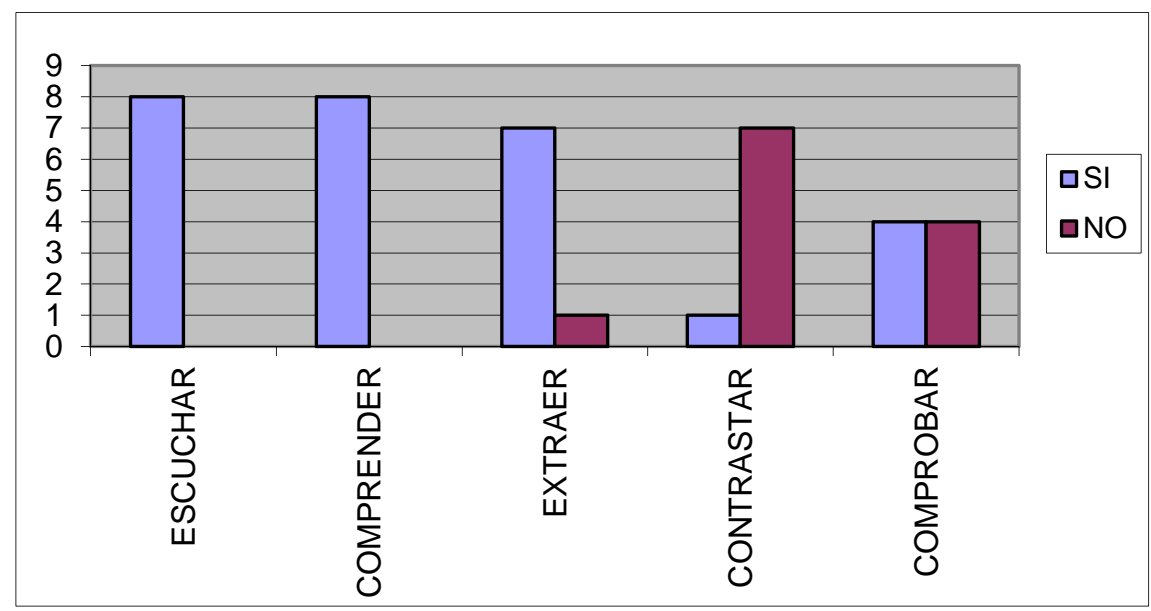

Figura 9: Procesos cognitivos implicados en la destreza Listening mediante TIC $\left(3^{\circ}\right.$ Ciclo $)$.

A continuación nos centraremos en la destreza reading en este ciclo (Figura 10). La mitad de los encuestados reconoce utilizar en sus aulas la lectura en soporte 
digital y no sólo en papel tradicional. Los equipos informáticos más utilizados en este ciclo para la lectura son los PC de casa, con un 50\%, y que incluso supone un mayor uso que en el propio centro, que sólo representa el $28 \%$ de los encuestados, lo que va demostrando una mayor autonomía por parte de los alumnos y manejo de las destrezas básicas del ordenador. En relación a las pizarras digitales sólo un 12\% lo hace con este fin.

En este ciclo nos llama la atención la introducción de un elemento innovador para la destreza de reading y es el utilizar las TIC para fomentar el hábito lector, propuesto por el Currículo. Los datos demuestran que sólo un $12 \%$ de los encuestados cumplen con esta finalidad. En este sentido un 37\% de los encuestados se pronuncia alegando que no ve posibilidad de fomentar el hábito lector a partir de las TIC, y más en concreto en el aula de lenguas extranjeras, aunque en mayoría son más los profesores que sí creen en este aspecto (62\%).

En relación a la destreza writing, un $62 \%$ del profesorado reconoce utilizar las TIC en esta destreza. Del mismo modo, también un $62 \%$ afirma que sus propios alumnos también las utilizan para elaborar textos escritos en lengua extranjera.

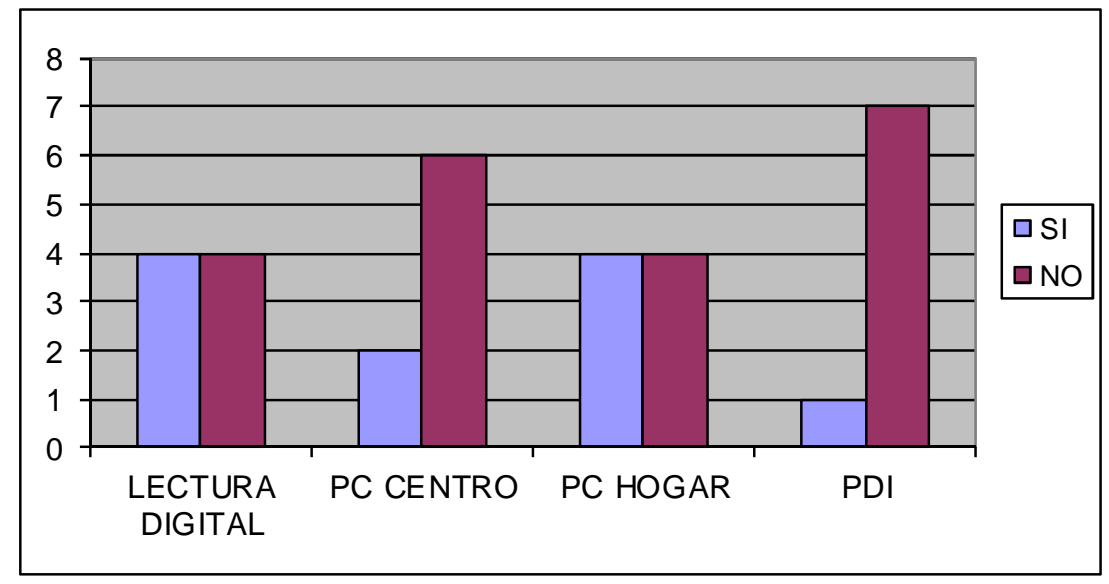

Figura 10: Soportes de lectura digital $\left(3^{\circ}\right.$ Ciclo $)$.

Respecto al uso de las TIC para el refuerzo de la lengua extranjera, un 63\% del profesorado reconoce usarlas dentro del aula y también para que sus alumnos lo consigan de forma autónoma. Pero los datos son más negativos cuando hablamos de ampliar el conocimiento de la lengua extranjera, el 50\% de los profesores no lo hace dentro del aula, ni el $63 \%$ fomenta su uso por su alumnado de forma autónoma.

El Currículo se muestra muy explícito al hablar de las TIC como medio para comunicarse con otros hablantes en la lengua extranjera pero todos los encuestados por unanimidad han respondido que no lo han llevado a la práctica. Además el 
Currículo también hace hincapié en que los alumnos muestren interés por este tipo de comunicación, pero a juicio de un $62 \%$ de los profesores implicados esto no es así.

Hemos utilizado una tabla de contingencia (Figura 11) para reflejar la frecuencia con la que los docentes encuestados utilizan las TIC en su labor en cada uno de los ciclos educativos. Esta variable se midió utilizando la escala de Likert con un rango de valores entre 1 y 5 , donde 1 equivale a muy frecuentemente y 5 a nada. Por tanto, cuánto más cerca esté del número cinco más se aleja de un uso frecuente de las TIC.

Estadísticos descriptivos

\begin{tabular}{|l|r|r|r|r|r|}
\hline & N & \multicolumn{1}{|c|}{ Mínimo } & \multicolumn{1}{c|}{ Máximo } & \multicolumn{1}{c|}{ Media } & Desv. típ. \\
\hline $\begin{array}{l}\text { Frecuencia en primer } \\
\text { ciclo }\end{array}$ & 11 & 1 & 4 & 2,73 &, 905 \\
$\begin{array}{l}\text { Frecuencia en segundo } \\
\text { ciclo }\end{array}$ & 13 & 1 & 4 & 2,69 &, 947 \\
Frecuencia en tercer ciclo & 8 & 2 & 4 & 2,88 &, 641 \\
N válido (según lista) & 3 & & & & \\
\hline
\end{tabular}

Figura 11: Tabla de contingencia para la variable Frecuencia de uso de las TIC.

\section{CONCLUSIONES Y DISCUSIÓN}

La incorporación de las TIC en la enseñanza del idioma «constituye un recurso mediador del aprendizaje y motivación para practicar y usar el idioma en situaciones reales, además de ayudar en la mejora de las habilidades lingüísticas de una manera interactiva y colaborativa» (Chacón y Pérez, 2011, p. 42). Sin embargo, para asumir que esto es así, deberíamos plantear la necesidad de que el profesorado conozca el Currículo oficial, y planifique a partir de él, desarrollando además la competencia de diseño de situaciones de aprendizaje y recursos didácticos que todos los maestros deberían tener. Esta es la primera de las deficiencias que hemos detectado en los resultados de esta investigación, y por tanto, el primer aspecto al que habría que prestar atención en los planes formativos iniciales y continuos destinados al profesorado.

Por otro lado, hemos podido apreciar que no hay grandes deficiencias en el uso que hacen los maestros de nuestra investigación que imparten docencia en primer ciclo y el uso que de las TIC propone para los niveles educativos incluidos en este. Pero también hemos de reconocer que en este ciclo el Currículo es menos exigente y sólo habla de las TIC al referirse al soporte en el que deben ir las audiciones que realizan los alumnos. El paso del tiempo ha ido dejando atrás soportes como las cintas tradicionales, que prácticamente ya no se utilizan, para dar paso a otros soportes más actuales como los $\mathrm{CD}$ y en algunos casos los DVD, y los más recientes recursos multimedia audiovisuales como los podcast (Sánchez y Solano, 2010; Chacón y Clevía, 2011). Además, los docentes de nuestro estudio cumplen unánimemente con el objetivo de que los alumnos comprendan los textos orales en lugar de repetirlos. En cambio, un aspecto en el que sí que discrepan los docentes con los 
postulados del Currículo es en el modo en el que introducen las nuevas tecnologías en este ciclo, que según el Currículo debe hacerse de forma progresiva. Pocos docentes reconocen hacerlo de esta manera. Esto nos lleva a cuestionarnos que quizás se deba a que no están incluidas dentro de su planificación docente, sino que recurren a las TIC de forma aleatoria e indiscriminada, sin realizar una reflexión crítica sobre su integración curricular, seleccionando la herramientas sin tener en cuenta previamente la tarea a desarrollar, como propone el modelo Tpack (Harris y Hofer, 2009; Koehler y Mishra, 2009). En este caso, se aprecia una deficiencia fundamental teniendo en cuenta su formación pedagógica, pues perciben las TIC más como un fin más que como un medio.

En el segundo ciclo al trabajar la destreza listening lo hacen conforme a lo establecido en el Currículo ya que la mayoría utiliza soportes multimedia para estas actividades. Además, cumplen con la finalidad de extraer información para realizar la tarea a partir de soportes multimedia. Sin embargo, cuando nos centramos en la lectura comprobamos que hay divergencias con el Currículo, ya que éste propone el uso de diferentes soportes digitales y, como hemos podido comprobar, se utilizan pocos soportes digitales para este fin, salvo los ofrecidos por las editoriales.

Otra área que sigue siendo deficitaria en este ciclo con relación al Currículo es el de utilizar las TIC para la escritura. El Currículo propone que los alumnos utilicen las TIC para escribir información, para transmitir información y para comunicarse, pero vemos que para escribir sólo algo más de la mitad de los maestros lo utiliza, mientras que el resultado es más pesimista cuando se trata de transmitir información o de comunicarse. Se echa en falta por tanto creatividad y capacidad de innovación para que los docentes planteen situaciones de aprendizaje auténticas y diseñen recursos didácticos adaptados al contexto y a los fines.

Deficitario con respecto a los postulados del Currículo es también el uso que hacen los maestros del segundo ciclo de las TIC para favorecer el aprendizaje autónomo de los alumnos y que estos amplíen y refuercen la lengua extranjera. Vemos que se le presta muy poca atención y que son pocos los que lo llevan a cabo, por lo que habría que plantear estrategias didácticas idóneas para que pudieran llevarse a cabo y de este modo cumplir con este principio que está establecido en el Currículo, entre las que podríamos destacar la Webquest, la Caza del Tesoro, así como metodologías activas y constructivistas como el Aprendizaje Basado en Tareas.

Todo esto nos lleva la conclusión de que en este ciclo no se está haciendo un uso correcto de las TIC si nos atenemos al Currículo. Además, no se cumple con los criterios de evaluación establecidos para este ciclo.

En el tercer ciclo también hemos apreciado deficiencias con respecto al Currículo. Los procesos cognitivos de contrastar y comprobar en la realización de actividades de listening son muy poco tenidas en cuenta por los maestros de nuestro estudio, por lo que habrá que incidir en la formación del profesorado para que adquieran estrategias didácticas para llevar a cabo actividades de este tipo con éxito. 
También nos ha llamado la atención el poco uso que se hace de soportes digitales para la lectura en tercer ciclo. Pero es más destacable aún el hecho de que no se esté fomentando el hábito lector entre los alumnos mediante las TIC.

En este ciclo se ha aumentado el nivel de uso de las TIC para que los alumnos amplíen y refuercen el uso de la lengua extranjera, comparando los datos con el ciclo anterior, pero todavía nos siguen pareciendo insuficientes. Hay que aprovechar la oportunidad de que los alumnos de este ciclo ya están más familiarizados con el uso de Internet y del PC, y por tanto, una actuación más sistemática a este respecto sería muy eficaz, es decir, todavía pensamos que están infravalorados.

Hay un aspecto muy novedoso en el Currículo para este ciclo con respecto a la función que propone para las TIC y es el de favorecer la comunicación con otros hablantes en lengua inglesa y además el que los alumnos muestren interés por establecer esta comunicación. En cambio, ninguno de los docentes de nuestro estudio lo lleva a la práctica. Quizás el motivo se deba a las propias infraestructuras del centro y a las características propias que tiene este tipo de comunicación, aunque la base del problema puede estar también en el desconocimiento de estrategias didácticas apoyadas en la interacción social, la colaboración y la comunicación, como las propuestas por Harris (1995).

Para finalizar este artículo diremos que tampoco nos parece acertada la frecuencia con la que recurren a las TIC los docentes de nuestra investigación, ya que en todos los ciclos están más cerca de «algunas veces», que el de «frecuentemente», Especialmente negativo nos parece que sea el tercer ciclo el que acuda con menos frecuencia a ellas, justo cuando los alumnos están más preparados para utilizarlas. No obstante, tal y como pronosticaba Hong (2010), también habrá que analizar los factores contextuales que influyen en la integración de las TIC en el aula a gran escala, al igual que defendía analizar si los maestros rodeados de un ambiente más propicio para el uso de las TIC las usan más frecuentemente. Consideramos que las TIC, bien llevadas, ofrecen muchas oportunidades de trabajar con ellas de forma eficaz y sin mucho esfuerzo, salvo el requerido para la formación del docente.

\section{REFERENCIAS BIBLIOGRÁFICAS}

AREA, Manuel. (2007a). Algunos principios para el desarrollo de buenas prácticas pedagógicas con las TICs en el aula. Comunicación y pedagogía, 222, 42-47.

AREA, Manuel. (2007b). Las tecnologías digitales y la innovación pedagógica en la Educación escolar (45-71). En ROMERO GRANADOS, Santiago. (dir). Introducción temprana a las TIC: estrategias para educar en un uso responsable en Educación Infantil y Primaria. Madrid: Ministerio de Educación Ciencia.

BEATTY, Ken (2003). Teaching and researching Computer-assisted Language Learning. London: Pearson Education.

CABERO, Julio. (2004). No todo es Internet: los medios audiovisuales e informáticos como recursos didácticos: Comunicación y Pedagogía, 200, 19-23. 
CACHÓN, Carmen \& PÉREZ, Clevia M.A. (2011). El podcast como innovación en la enseñanza del inglés como lengua extranjera. Revista Pixel-Bit de medios y Educación, 39, 41-54.

CLARK, Richard. \& SALOMON, Gabriel. (1986). Media in Teaching (464-478). En WITTROCK, Merlin. (Ed). Handbook of research on teaching. New York. MacMillan Reference Books.

CLARK, Richard. (2001). Media are te vehicles. The Opening Arguments. En Clark, R.E. (Ed). Learning from media: arguments, analysis, and evidence. Connecticut: Information Age Publishing.

CUBAN, Larry. (1986). Teachers and Machines: The classroom use of Technology Since 1920. New York: Teachers College Press. CUBAN.

CURRAN, Phillip., DEGUENT, Sandrine., LUND, Sian.W., MILETTO, Heather. \& VAN DER STRAETEN, Carla. (2000). Methodology in language learning TKit. Council of European Commission. Council of European publishing. http://youth-partnership-eu.coe.int/youthpartnership/documents/Publications/T_kits/2/tkit2.pdf [Consulta: 10 enero de 2013].

DELFIN, M., DETTORI, G. \& LUPI, V. (2009). Task-based learning and ICET: creative activities in the context of a European Project. eLearning Papers, 16. http://www.elearningeuropa.info/files/media/media20253.pdf [Consulta: 10 enero de 2013].

DESJARDINS, F. \& PETERS, M. (2007). SINGLE COURSE APPROACH versus a program approach to develop technological competence in pre-service language teachers (3-21). En KASSEN, M. A., LAVINE, R., MURPHY-JUDY, K. \& PETERS, M. (eds). (2007). Preparing and developing technology-proficient L2 teachers. San Marcos: CALICO.

ELLIS, R. (2003). Task-Based language Learning and Teaching. Oxford: Oxford University Press.

HARRIS, Judi. (1995). Organizing and Facilitating Tellecolaborative Projects. The Computing Teacher, 22, 5. http://lrs.ed.uiuc.edu/mining/February95-TCT.html [Consulta: 10 enero de 2013].

HARRIS, Judi., \& HOFER, Mark. (2009). Instructional Maddux, C. D (Ed.). Research highlights in technology and teacher education 2009 (99-108). Chesapeake, VA: Society for Information Technology inn Teacher Education (SITE).

HEGELHEIMER, Volker. (2006). When the technology course is required (117133). En Hubbard, P. \& Levy, M. (eds) (2006). Teacher education in CALL. Philadelphia: John Benjamins Publishing Company.

HONG, K.H. (2010). CALL teacher education as an impetus for L2 teacher in integrating technology. ReCALL, 22 (1): 53-69. 
HUBBARD, P. \& LEVY, M. (eds) (2006). En Hubbard, P. \& Levy, M. (eds) (2006). Teacher education in CALL. Philadelphia: John Benjamins Publishing Company.

KASSEN, M.A., LAVINE, R., MURPHY-JUDY, K. \& PETERS, M. (2007). Preparing and developing technology-proficient L2 teachers. San Marcos: CALICO.

KESSLER, G. (2006). Assessing CALL teacher training: What are we doing and what could we do better (23-42). En Hubbard, P. \& Levy, M. (eds) (2006). Teacher education in CALL. Philadelphia: John Benjamins Publishing Company.

KOEHLER, Matthew. J., \& MISHRA, Punya. (2009). What is technological pedagogical content knowledge? Contemporary Issues in Technology and Teacher Education, 9(1), 60-70.

LUKE, Christopher. \& BRITTEN, Jody. (2007). The expanding role of technology in foreign language teacher education programs. CALICO Journal, 24 (2): 253267.

MARTÍN LÓPEZ, Elena. El enfoque por tareas y la didáctica en lengua inglesa en el nuevo Grado de Magisterio. Revista Didáctica. Lengua y Literatura, 21, 2555-279.

MARTÍNEZ SÁNCHEZ, Francisco. (2007). La integración escolar de las nuevas tecnologías (21-40). En CABERO, Julio. (Coord) (2007). Nuevas Tecnologías aplicadas a la Educación. Madrid: McGrawHill.

SOLANO, Isabel M. (2010). Estrategias metodológicas para el uso de las redes en Educaión Infantil. http://digitum.um.es/xmlui/bitstream/10201/13627/8/Estrategias_TIC_Ed_Infan til.pdf [Consulta: 10 enero de 2013].

SOLÍS, Juan. y MACÍAS, Carmen. (2009). Lengua inglesa y TICs en el aula: un análisis de percepciones formativas y necesidades docentes. En ATRIO, S. y BAUTISTA, A. Actas del Congreso Internacional sobre Antropología Audiovisual e Investigación en Tecnología Educativa. Madrid: Rute Ediciones.

TOCHON, F. \& BLACK, N.J. (2007). Narrative analysis of electronic portfolios: Preservice teacher's struggles in researching Pedagogically Appropriate Technology Integration (PATRI) (295-320). En KASSEN, M.A., LAVINE, R., MURPHY-JUDY, K. \& PETERS, M. (2007). Preparing and developing technology-proficient L2 teachers. San Marcos: CALICO.

WARSCHAUER, Mark. \& HEALEY, Deborah. (1998). Computers and language learning: An overview. Language teaching, 31, 57-71. 